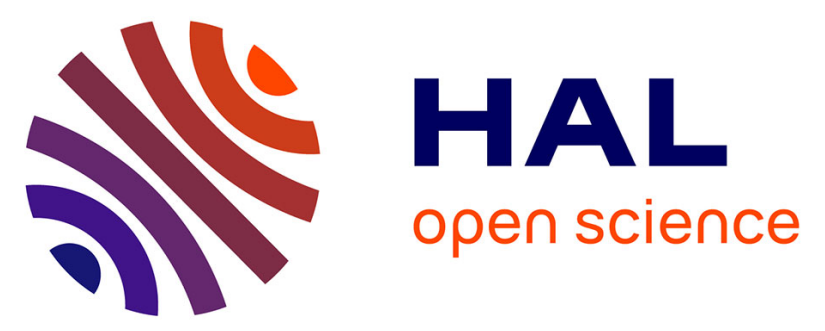

\title{
Ferrocenyl compounds possessing protected phenol and thiophenol groups: Synthesis, X-ray structure, and in vitro biological effects against breast cancer
}

Julia B. Heilmann, Elisabeth A. Hillard, Marie-Aude Plamont, Pascal Pigeon, Michael Bolte, Gérard Jaouen, Anne Vessières

\section{To cite this version:}

Julia B. Heilmann, Elisabeth A. Hillard, Marie-Aude Plamont, Pascal Pigeon, Michael Bolte, et al.. Ferrocenyl compounds possessing protected phenol and thiophenol groups: Synthesis, X-ray structure, and in vitro biological effects against breast cancer. Journal of Organometallic Chemistry, 2008, 693 (8-9), pp.1716-1722. 10.1016/j.jorganchem.2007.12.011 . hal-01230397

\section{HAL Id: hal-01230397 \\ https://hal.science/hal-01230397}

Submitted on 22 May 2021

HAL is a multi-disciplinary open access archive for the deposit and dissemination of scientific research documents, whether they are published or not. The documents may come from teaching and research institutions in France or abroad, or from public or private research centers.
L'archive ouverte pluridisciplinaire HAL, est destinée au dépôt et à la diffusion de documents scientifiques de niveau recherche, publiés ou non, émanant des établissements d'enseignement et de recherche français ou étrangers, des laboratoires publics ou privés. 


\title{
Ferrocenyl compounds possessing protected phenol and thiophenol groups: Synthesis, $\mathrm{X}$-ray structure, and in vitro biological effects against breast cancer
}

Julia B. Heilmann ${ }^{\text {a }}$, Elizabeth A. Hillard ${ }^{\text {a }}$, Marie-Aude Plamont ${ }^{\text {a }}$, Pascal Pigeon ${ }^{\text {a }}$, Michael Bolte $^{\mathrm{b}}$, Gérard Jaouen ${ }^{\mathrm{a}}$, Anne Vessières ${ }^{\mathrm{a}, *}$

${ }^{\text {a }}$ Laboratoire de Chimie et Biochimie des Complexes Moléculaires, UMR CNRS 7576, Ecole Nationale Supérieure de Chimie de Paris, 11 rue Pierre et Marie Curie, 75231 Paris Cedex 05, France

${ }^{\mathrm{b}}$ Institut für Anorganische Chemie, J.W. Goethe-Universität Frankfurt, Max von Laue-Strasse 7, D-60438 Frankfurt (Main), Germany

* Corresponding author. Tel.: +33 01442767 29; fax: +33 0143260061 . E-mail address: a-vessieres@enscp.fr (A. Vessières).

Keywords : Ferrocene; Breast cancer; Bioorganometallic chemistry; Iron

\begin{abstract}
We have previously shown that conjugated ferrocenyl $p$-phenols show strong cytotoxic effects against both the hormone-dependent MCF-7 and hormone-independent MDA-MB-231 breast cancer cell lines, possibly via metabolic quinone methide (QM) formation. To further evaluate this proposed mechanism, we have created a series of ferrocenyl prodrugs containing methyl and acetyl-protected thio- and oxo-phenols: 2-ferrocenyl-1,1-bis(4-acetoxyphenyl)-but-1-ene (5), 2-ferrocenyl-1,1-bis(4-thioacetylphenyl)-but-1-ene (6), 2-ferrocenyl-1,1-bis(4methoxyphenyl)-but-1-ene (7), and 2-ferrocenyl-1,1-bis(4-thiomethylphenyl)-but-1-ene (8), which might be activated by hydrolysis enzymes in situ. Only the acetoxy $\mathbf{5}$ displayed antiproliferative effects $\left(\mathrm{IC}_{50}\right.$ on MDA-MB-231 of $0.5 \mu \mathrm{M}$ ) while 6-8 act as pure estrogens (proliferative on MCF-7 and little to no effect on MDA-MB-231). The behaviour of 5 is
\end{abstract}


similar to that previously found for the free phenol 2-ferrocenyl-1,1-di(4-hydroxyphenyl)-but1-ene (2), indicating that 5 is metabolized in situ to $\mathbf{2}$, which could then undergo oxidative QM formation. The observation that the thioacetyl 6 is not cytotoxic suggests that the in situ oxidative chemistry of the putative ferrocenyl thiophenol is different from that of $\mathbf{2}$. Because $p$-thioquinone methides are practically unknown, the negative results for $\mathbf{6}$ further implicate the bioformation of the QM in the case of $\mathbf{2}$ and related compounds. The lack of cytotoxicity of $\mathbf{7}$ and $\mathbf{8}$ can be attributed to lack of efficient hydrolysis in situ. Estrogen receptor binding affinity studies for the compounds and the X-ray structure of $\mathbf{8}$ are also reported.

\section{Introduction}

Bioorganometallic chemistry is a growing multidisciplinary field which encompasses the synthesis and study of organometallic complexes of biological importance [1]. One of the most promising applications of bioorganometallic chemistry is in the area of drug development. Ferroquine, a ferrocenyl analogue of chloroquine, has shown activity against chloroquine-resistant strains of malaria, and is currently in phase II clinical trials with SanofiAventis [2], while two ruthenium arene complexes have entered clinical trials as antimetastatic candidates [3].

Our work is focused on the use of organometallic nanovectors in oncology, especially cancers of the breast and prostate [4-7]. We have found that compounds possessing a ferrocenyl moiety linked to a $p$-phenol group by a conjugated system show considerable potency against breast cancer cells, with $\mathrm{IC}_{50}$ values ranging from 0.44 to $1.1 \mu \mathrm{M}$ against the hormoneindependent MDA-MB-231 cell line, Chart 1. We have posited that these compounds can be activated by in situ oxidation to form a cytotoxic quinone methide (QM) species. Electrochemical experiments have suggested that this transformation can occur at low potentials, due to the role of the ferricenium cation as an intramolecular oxidant of the phenol group in basic conditions [8-10]. We now wish to understand how the replacement of the oxygen atom by a sulfur atom will influence the cytotoxicity of these types of molecules. 


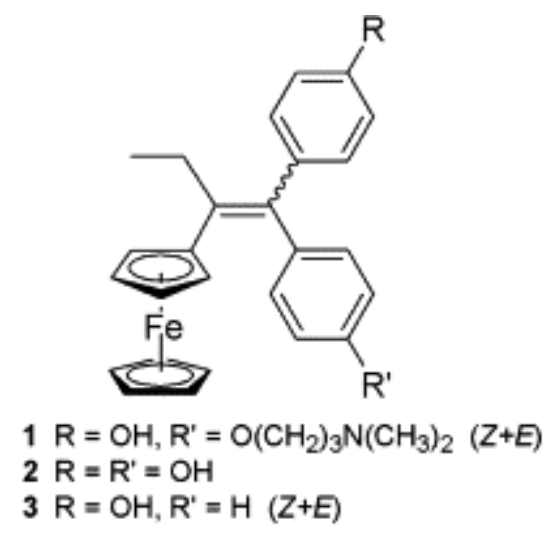

Chart 1. Previously reported cytotoxic ferrocenyl phenolic compounds 1 [6], 2 [11], and 3 [5].

The formation of QMs is an important pathway for the metabolism of many compounds, including natural products [12-14], selective estrogen receptor modulators [15-18], and drugs [19-22]. However, to our knowledge, only one example of an isolable $p$-thioquinone methide has been reported, 10-(dicyanomethylene)thioanthrone, which is stabilized by electron withdrawing cyano groups and an annelated phenyl ring [23]. Thioquinone methides do not seem to be important in biology, because oxidation of aromatic thiols immediately leads to dimerization or reaction with other thiols, thiolates, or molecular oxygen [24]. Therefore, if QM formation is indeed an important mechanism of cytotoxicity in the series of ferrocenyl phenol compounds, one would expect their sulfur congeners to be non-cytotoxic.

However, other mechanisms of cytotoxicity could also be relevant. It should be mentioned that phenolic compounds [25] and ferricenium salts [26] can produce reactive oxygen species through phenol-quinone redox cycling and Fenton-Haber-Weiss reactions, leading to cell damage. The oxidation of aromatic thiols has also been shown to generate $\mathrm{H}_{2} \mathrm{O}_{2}$ and provoke oxidative stress [24,27]. Therefore, if the cytotoxicity observed in ferrocenyl phenols arises mainly through oxidative stress instead of QM formation, the substitution of the oxygen atom by a sulfur atom would be expected to retain the compounds' ability to undergo redox cycling, Fenton chemistry, and observed cytotoxic properties.

We have synthesized both oxo- and thio-analogues of compound 2, protected by methyl or acetyl groups, Chart 2, and tested their effects on the proliferation of the estrogen receptor positive MCF-7 and estrogen receptor negative MDA-MB-231 cell line. Protecting groups are often used in drug formulation to enhance the stability and bioavailability of molecules $[28,29]$. The protected compounds can be considered as prodrugs; only after hydrolysis by 
appropriate enzymes is the active drug released. In terms of this study, the use of two different protecting groups is expected to provide information about the hydrolysis of these compounds to the free phenol or thiophenol in vitro. Finally, we were also interested in determining the influence that the change from oxygen to sulfur has on estrogen receptor binding, and report here the affinity for the estrogen receptors $\alpha$ and $\beta$.

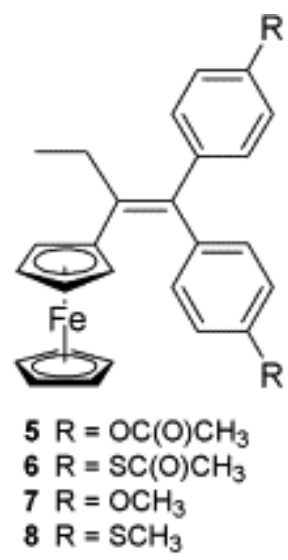

Chart 2. New compounds 5-8. Compound $\mathbf{4}$ is a reaction intermediate that was isolated and chemically characterized, although biological results are not reported here.

\section{Experimental}

\subsection{General remarks}

The synthesis of all compounds was carried out using standard Schlenk techniques, under an argon atmosphere. THF and diethyl ether were dried and distilled over sodium/benzophenone, while $\mathrm{CH}_{2} \mathrm{Cl}_{2}$ was dried and distilled over $\mathrm{CaH}_{2}$, prior to use. Thin layer and column chromatography was performed on silica gel $60 \mathrm{GF} 254$ and $60 \mathrm{M}$, respectively. ${ }^{1} \mathrm{H}$ and ${ }^{13} \mathrm{C}$ NMR spectra were acquired on a Bruker $300 \mathrm{MHz}$ spectrometer. EI-MS was performed on a Nermag R 10-10C spectrometer; ESI-MS using a triple quadrupole mass spectrometer API 3000 LC-MS/MS system (Applied Biosystems, PE Sciex) in positive-ion mode; and high resolution mass spectrometry (HRMS) with a Joel MS 7000 instrument. Elemental analyses were performed at the microanalysis laboratory of the CNRS (Gif sur Yvette, France). Melting points were measured with a Kofler device.

4-Bromothioanisole, 4-bromothiophenol and 4,4-dimethoxybenzophenone were purchased from Aldrich and used without further purification. (4-Bromo)phenyl-t-butylthioether [30], 
propionyl ferrocene [31], 4,4'-bis(t-butylthio)benzophenone [32], and 4,4'bis(methylthio)benzophenone [32] were synthesized according to procedures found in the literature.

\subsection{2-Ferrocenyl-1,1'-bis(4-thio-t-butylphenyl)-but-1-ene, 4}

$\mathrm{TiCl}_{4}(3.35 \mathrm{mmol}, 0.636 \mathrm{~g})$ was added dropwise to a slurry of zinc dust $(5.02 \mathrm{mmol}, 0.328 \mathrm{~g})$ in THF (15 ml). The resulting black mixture was heated under reflux for $2 \mathrm{~h}$ and then cooled to r.t. Propionyl ferrocene (1.42 mmol, $0.344 \mathrm{~g})$ and 4,4'-bis(t-butylthio)benzophenone (0.837 mmol, $0.300 \mathrm{~g}$ ) were dissolved in $\mathrm{THF}(15 \mathrm{ml})$ and added to the $\mathrm{Zn} / \mathrm{TiCl}_{4}$ mixture. The reaction mixture was heated under reflux for $5 \mathrm{~h}$ (reaction monitored by TLC). After cooling to r.t. the mixture was poured onto $\mathrm{H}_{2} \mathrm{O} / \mathrm{HCl} / \mathrm{ice}$, whereupon the colour changed from dark brown to orange. The aqueous phase was extracted with $\mathrm{CH}_{2} \mathrm{Cl}_{2}(4 \times 25 \mathrm{ml})$ and the combined organic layers were washed with $\mathrm{H}_{2} \mathrm{O}(3 \times 20 \mathrm{ml})$, dried over $\mathrm{MgSO}_{4}$, and concentrated. Purification by flash chromatography $\left(\mathrm{CH}_{2} \mathrm{Cl}_{2} /\right.$ hexane $\left.1: 1\right)$ yielded the pure product as a red solid $(120 \mathrm{mg}, 25 \%) .{ }^{1} \mathrm{H} \mathrm{NMR}\left(300 \mathrm{MHz}, \mathrm{CDCl}_{3}\right): \delta=7.50(\mathrm{~d}, J=8.1 \mathrm{~Hz}$, $\left.2 \mathrm{H}, \mathrm{C}_{6} H_{4}\right), 7.37\left(\mathrm{~d}, J=8.1 \mathrm{~Hz}, 2 \mathrm{H}, \mathrm{C}_{6} H_{4}\right), 7.19\left(\mathrm{~d}, J=8.1 \mathrm{~Hz}, 2 \mathrm{H}, \mathrm{C}_{6} H_{4}\right), 7.02(\mathrm{~d}, J=8.1 \mathrm{~Hz}$, $\left.2 \mathrm{H}, \mathrm{C}_{6} H_{4}\right), 4.12\left(\mathrm{~s}, 5 \mathrm{H}, \mathrm{C}_{5} H_{5}\right), 4.08\left(\mathrm{t}, J=1.8 \mathrm{~Hz}, 2 \mathrm{H}, \mathrm{C}_{5} H_{4}\right), 3.86\left(\mathrm{t}, J=1.8 \mathrm{~Hz}, 2 \mathrm{H}, \mathrm{C}_{5} H_{4}\right)$, $2.60\left(\mathrm{q}, J=7.5 \mathrm{~Hz}, 2 \mathrm{H}, \mathrm{CH}_{2}\right), 1.31\left(\mathrm{~s}, 9 \mathrm{H}, \mathrm{C}\left(\mathrm{CH}_{3}\right)_{3}\right), 1.27\left(\mathrm{~s}, 9 \mathrm{H}, \mathrm{C}\left(\mathrm{CH}_{3}\right)_{3}\right), 1.01(\mathrm{t}$, $\left.J=7.5 \mathrm{~Hz}, 3 \mathrm{H}, \mathrm{CH}_{3}\right) .{ }^{13} \mathrm{C} \mathrm{NMR}\left(75 \mathrm{MHz}, \mathrm{CDCl}_{3}\right): \delta=144.9,144.5,138.9\left(\mathrm{C}^{\text {quat }}\right), 137.3$, 137.2 $\left(C_{6} \mathrm{H}_{4}\right), 136.5,130.6,130.4\left(\mathrm{C}^{\text {quat }}\right), 130.1,129.5\left(C_{6} \mathrm{H}_{4}\right), 86.5\left(\mathrm{Fc}^{i p s o}\right), 69.3\left(C_{5} H_{4}\right), 69.2$ $\left(C_{5} \mathrm{H}_{5}\right), 68.2\left(C_{5} \mathrm{H}_{4}\right), 45.8,45.7\left(C\left(\mathrm{CH}_{3}\right)_{3}\right), 31.0,30.9\left(\mathrm{C}\left(\mathrm{CH}_{3}\right)_{3}\right), 28.1\left(\mathrm{CH}_{2}\right), 15.3\left(\mathrm{CH}_{3}\right) . \mathrm{MS}$ (EI, $70 \mathrm{eV}$ ) m/z: $568[\mathrm{M}]^{+}, 121[\mathrm{CpFe}]^{+}$. HRMS calcd for $\mathrm{C}_{34} \mathrm{H}_{40} \mathrm{FeS}_{2}:$ 568.1921; found: 568.1929 [M] ${ }^{+}$. Elemental Anal. Calc. for $\mathrm{C}_{34} \mathrm{H}_{40} \mathrm{FeS}_{2}$ : C, 71.81; H, 7.09; S, 11.28. Found: C, 71.80; H, 7.01; S, 11.31. m.p. $=150^{\circ} \mathrm{C}$.

\subsection{2-Ferrocenyl-1,1-bis(4-acetoxyphenyl)-but-1-ene, 5}

Acetyl chloride $(1 \mathrm{ml})$ was added dropwise to a suspension of $2(0.471 \mathrm{mmol}, 0.200 \mathrm{~g})$ in $\mathrm{CH}_{2} \mathrm{Cl}_{2}(15 \mathrm{ml})$ at r.t. The reaction mixture was stirred at r.t. overnight, yielding a clear orange solution. The solution was then poured into $\mathrm{H}_{2} \mathrm{O}(20 \mathrm{ml})$ and the phases were separated. The aqueous phase was extracted with $\mathrm{CH}_{2} \mathrm{Cl}_{2}(3 \times 20 \mathrm{ml})$ and the combined organic layers were washed with $\mathrm{H}_{2} \mathrm{O}(2 \times 20 \mathrm{ml})$, dried over $\mathrm{MgSO}_{4}$, and concentrated. Flash chromatography $\left(\mathrm{CH}_{2} \mathrm{Cl}_{2}\right)$ yielded the pure product as an orange solid $(216 \mathrm{mg}, 90 \%) .{ }^{1} \mathrm{H}$ NMR $\left(300 \mathrm{MHz}, \mathrm{CDCl}_{3}\right): \delta=7.20\left(\mathrm{~d}, J=8.7 \mathrm{~Hz}, 2 \mathrm{H}, \mathrm{C}_{6} H_{4}\right), 7.06\left(\mathrm{~d}, J=8.7 \mathrm{~Hz}, 2 \mathrm{H}, \mathrm{C}_{6} H_{4}\right)$, $7.05\left(\mathrm{~d}, J=8.7 \mathrm{~Hz}, 2 \mathrm{H}, \mathrm{C}_{6} H_{4}\right), 6.94\left(\mathrm{~d}, J=8.7 \mathrm{~Hz}, 2 \mathrm{H}, \mathrm{C}_{6} H_{4}\right), 4.11\left(\mathrm{~s}, 5 \mathrm{H}, \mathrm{C}_{5} H_{5}\right), 4.10$ (t, $\left.J=1.8 \mathrm{~Hz}, 2 \mathrm{H}, \mathrm{C}_{5} H_{4}\right), 3.90\left(\mathrm{t}, J=1.8 \mathrm{~Hz}, 2 \mathrm{H}, \mathrm{C}_{5} H_{4}\right), 2.59\left(\mathrm{q}, J=7.5 \mathrm{~Hz}, 2 \mathrm{H}, \mathrm{CH}_{2}\right), 2.30(\mathrm{~s}$, 
$\left.3 \mathrm{H}, \mathrm{C}(\mathrm{O}) \mathrm{CH}_{3}\right), 2.27\left(\mathrm{~s}, 3 \mathrm{H}, \mathrm{C}(\mathrm{O}) \mathrm{CH}_{3}\right), 1.03\left(\mathrm{t}, J=7.5 \mathrm{~Hz}, 3 \mathrm{H}, \mathrm{CH}_{3}\right) .{ }^{13} \mathrm{C} \mathrm{NMR}(75 \mathrm{MHz}$, $\left.\mathrm{CDCl}_{3}\right): \delta=169.3,169.2(C=\mathrm{O}), 149.0,149.0,141.8,141.6,138.6,135.9\left(\mathrm{C}^{\text {quat }}\right), 130.9$, 130.3, 121.3, $121.1\left(C_{6} \mathrm{H}_{4}\right), 86.3\left(\mathrm{Fc}^{i p s o}\right), 69.3\left(C_{5} \mathrm{H}_{4}\right), 69.1\left(C_{5} \mathrm{H}_{5}\right), 68.3\left(C_{5} \mathrm{H}_{4}\right), 28.0\left(C_{2}\right)$, $21.2\left(\mathrm{C}(\mathrm{O}) \mathrm{CH}_{3}\right), 15.4\left(\mathrm{CH}_{3}\right)$. MS (EI, $\left.70 \mathrm{eV}\right) \mathrm{m} / z: 508[\mathrm{M}]^{+}, 465\left[\mathrm{M}-\mathrm{CH}_{3} \mathrm{CO}\right]^{+}, 121[\mathrm{CpFe}]^{+}$, $43\left[\mathrm{CH}_{3} \mathrm{CO}\right]^{+}$. HRMS calcd for $\mathrm{C}_{30} \mathrm{H}_{28} \mathrm{FeO}_{4}$ : 508.1337; found 508.1337. m.p. $=119{ }^{\circ} \mathrm{C}$.

\subsection{2-Ferrocenyl-1,1-bis(4-thioacetylphenyl)-but-1-ene, 6}

Compound 4 (0.341 mmol, $0.194 \mathrm{~g})$ was dissolved in $\mathrm{CH}_{2} \mathrm{Cl}_{2}(25 \mathrm{ml})$ and acetyl chloride $(1.4 \mathrm{ml})$ was added at r.t. To the resulting mixture, a $1.0 \mathrm{M}$ solution of $\mathrm{BBr}_{3}$ in $\mathrm{CH}_{2} \mathrm{Cl}_{2}$ $(2.73 \mathrm{ml}, 2.73 \mathrm{mmol})$ was added and the dark brown solution was stirred at r.t. for 6 days. The mixture was poured into an aqueous solution of $\mathrm{NaHCO}_{3}$ and $\mathrm{CH}_{2} \mathrm{Cl}_{2}(20 \mathrm{ml})$ was added. The phases were separated and the aqueous phase was extracted with $\mathrm{CH}_{2} \mathrm{Cl}_{2}(4 \times 25 \mathrm{ml})$. The combined organic layers were washed with $\mathrm{H}_{2} \mathrm{O}(3 \times 20 \mathrm{ml})$, dried over $\mathrm{MgSO}_{4}$, and concentrated. Purification by flash chromatography $\left(\mathrm{CH}_{2} \mathrm{Cl}_{2}\right)$ yielded the pure product as a red oil (113 mg, 61\%). ${ }^{1} \mathrm{H}$ NMR (300 MHz, $\left.\mathrm{CDCl}_{3}\right): \delta=7.38$ (d, $\left.J=8.4 \mathrm{~Hz}, 2 \mathrm{H}, \mathrm{C}_{6} H_{4}\right), 7.27-$ $7.23\left(\mathrm{~m}, 4 \mathrm{H}, \mathrm{C}_{6} H_{4}\right), 7.09\left(\mathrm{~d}, J=8.4 \mathrm{~Hz}, 2 \mathrm{H}, \mathrm{C}_{6} H_{4}\right), 4.13-4.11\left(\mathrm{~m}, 7 \mathrm{H}, \mathrm{C}_{5} H_{4} / \mathrm{C}_{5} H_{5}\right), 3.91(\mathrm{t}$, $\left.J=1.8 \mathrm{~Hz}, 2 \mathrm{H}, \mathrm{C}_{5} H_{4}\right), 2.61\left(\mathrm{q}, J=7.5 \mathrm{~Hz}, 2 \mathrm{H}, \mathrm{CH}_{2}\right), 2.42\left(\mathrm{~s}, 3 \mathrm{H}, \mathrm{C}(\mathrm{O}) \mathrm{CH}_{3}\right), 2.39$ (s, 3H, $\left.\mathrm{C}(\mathrm{O}) \mathrm{CH}_{3}\right), 1.03\left(\mathrm{t}, J=7.5 \mathrm{~Hz}, 3 \mathrm{H}, \mathrm{CH}_{3}\right) .{ }^{13} \mathrm{C} \mathrm{NMR}\left(75 \mathrm{MHz}, \mathrm{CDCl}_{3}\right): \delta=194.1,194.0(\mathrm{C}=$ O), 145.3, 145.0, 139.7, $135.7\left(\mathrm{C}^{\text {quat }}\right), 134.2,134.1,130.9,130.3\left(C_{6} \mathrm{H}_{4}\right), 125.7,97.0\left(\mathrm{C}^{\text {quat }}\right)$, $86.1\left(\mathrm{Fc}^{i p s o}\right), 69.5\left(C_{5} \mathrm{H}_{4}\right), 69.2\left(C_{5} \mathrm{H}_{5}\right), 68.5\left(C_{5} \mathrm{H}_{4}\right), 30.2,30.1\left(\mathrm{C}(\mathrm{O}) C_{3}\right), 28.2\left(C_{2}\right), 15.5$ $\left(\mathrm{CH}_{3}\right)$. MS (ESI) $m / z$ : $540.3[\mathrm{M}]^{+}$. HRMS calcd for $\mathrm{C}_{30} \mathrm{H}_{28} \mathrm{FeO}_{2} \mathrm{~S}_{2}$ : 540.0880; found: $540.0880[\mathrm{M}]^{+.}$. m.p. $=111-113^{\circ} \mathrm{C}$.

\subsection{2-Ferrocenyl-1,1-bis(4-methoxyphenyl)-but-1-ene, 7}

Same procedure as 4 using $\mathrm{TiCl}_{4}(32.8 \mathrm{mmol}, 6.22 \mathrm{~g})$, zinc dust $(61.2 \mathrm{mmol}, 4.00 \mathrm{~g})$ in THF, propionyl ferrocene (10.0 mmol, $2.42 \mathrm{~g}$ ) and 4,4'-dimethoxybenzophenone (20.0 mmol, $4.84 \mathrm{~g})$. Yield $=3.890 \mathrm{~g}, 86 \%$. ${ }^{1} \mathrm{H}$ NMR $\left(300 \mathrm{MHz}, \mathrm{CDCl}_{3}\right): \delta=7.16(\mathrm{~d}, J=7.0 \mathrm{~Hz}, 2 \mathrm{H}$, $\left.\mathrm{C}_{6} H_{4}\right), 7.01\left(\mathrm{~d}, J=7.0 \mathrm{~Hz}, 2 \mathrm{H}, \mathrm{C}_{6} H_{4}\right), 6.90\left(\mathrm{~d}, J=7.0 \mathrm{~Hz}, 2 \mathrm{H}, \mathrm{C}_{6} H_{4}\right), 6.79$ (d, J=7.0 Hz, 2H, $\left.\mathrm{C}_{6} H_{4}\right), 4.15\left(\mathrm{~s}, 5 \mathrm{H}, \mathrm{C}_{5} H_{5}\right), 4.12\left(\mathrm{t}, J=1.9 \mathrm{~Hz}, 2 \mathrm{H}, \mathrm{C}_{5} H_{4}\right), 3.95\left(\mathrm{t}, J=1.9 \mathrm{~Hz}, 2 \mathrm{H}, \mathrm{C}_{5} H_{4}\right), 3.85$ (s, 3H, $\left.\mathrm{OCH}_{3}\right), 3.81\left(\mathrm{~s}, 3 \mathrm{H}, \mathrm{OCH}_{3}\right), 2.62\left(\mathrm{q}, J=7.4 \mathrm{~Hz}, 2 \mathrm{H}, \mathrm{CH}_{2}\right), 1.07$ (t, $J=7.4 \mathrm{~Hz}, 3 \mathrm{H}$, $\left.\mathrm{CH}_{3}\right) .{ }^{13} \mathrm{C} \mathrm{NMR}\left(75 \mathrm{MHz}, \mathrm{CDCl}_{3}\right): \delta=157.9,137.5,137.3,137.2,136.7$ (C quat $), 131.0,130.4$, 113.6, $113.5\left(C_{6} \mathrm{H}_{4}\right), 87.2\left(\mathrm{Fc}^{i p s o}\right), 69.3\left(C_{5} \mathrm{H}_{4}\right), 69.2\left(C_{5} \mathrm{H}_{5}\right), 68.0\left(C_{5} \mathrm{H}_{4}\right), 55.2,55.1\left(\mathrm{OCH}_{3}\right)$, $28.0\left(\mathrm{CH}_{2}\right), 15.5\left(\mathrm{CH}_{3}\right)$. MS (EI, $\left.70 \mathrm{eV}\right) \mathrm{m} / z: 452[\mathrm{M}]^{+}, 423[\mathrm{M}-\mathrm{Et}]^{+}, 387[\mathrm{M}-\mathrm{Cp}]^{+}, 121$ [FeCp] $]^{+}$Elemental Anal. Calc. for $\mathrm{C}_{28} \mathrm{H}_{28} \mathrm{FeO}_{2}$ : C, 74.34; H, 6.24. Found: C, 73.99; H, $6.26 \%$. m.p. $=94{ }^{\circ} \mathrm{C}$. 
This compound had previously been obtained by a different route [33]; this is the first communication of its characterization.

\subsection{2-Ferrocenyl-1,1-bis(4-thiomethylphenyl)-but-1-ene, 8}

Same procedure as 4 using $\mathrm{TiCl}_{4}(3.28 \mathrm{mmol}, 0.623 \mathrm{~g})$, zinc dust $(5.91 \mathrm{mmol}, 0.386 \mathrm{~g})$, propionyl ferrocene $(1.09 \mathrm{mmol}, \quad 0.265 \mathrm{~g})$ and 4, 4'-bis(methylthio)benzophenone $(0.729 \mathrm{mmol}, 0.200 \mathrm{~g})$. Yield $=106 \mathrm{mg}, 30 \% .{ }^{1} \mathrm{H}$ NMR $\left(300 \mathrm{MHz}, \mathrm{CDCl}_{3}\right): \delta=7.21(\mathrm{~d}$, $\left.J=8.4 \mathrm{~Hz}, 2 \mathrm{H}, \mathrm{C}_{6} H_{4}\right), 7.10\left(\mathrm{~d}, J=8.4 \mathrm{~Hz}, 4 \mathrm{H}, \mathrm{C}_{6} H_{4}\right), 6.96\left(\mathrm{~d}, J=8.4 \mathrm{~Hz}, 2 \mathrm{H}, \mathrm{C}_{6} H_{4}\right), 4.11$ (s, $\left.5 \mathrm{H}, \mathrm{C}_{5} H_{5}\right), 4.09\left(\mathrm{t}, J=1.8 \mathrm{~Hz}, 2 \mathrm{H}, \mathrm{C}_{5} H_{4}\right), 3.92\left(\mathrm{t}, J=1.8 \mathrm{~Hz}, 2 \mathrm{H}, \mathrm{C}_{5} H_{4}\right), 2.59$ (q, $J=7.5 \mathrm{~Hz}$, $\left.2 \mathrm{H}, \mathrm{CH}_{2}\right), 2.49\left(\mathrm{~s}, 3 \mathrm{H}, \mathrm{SCH}_{3}\right), 2.45\left(\mathrm{~s}, 3 \mathrm{H}, \mathrm{SCH}_{3}\right), 1.02\left(\mathrm{t}, J=7.5 \mathrm{~Hz}, 3 \mathrm{H}, \mathrm{CH}_{3}\right) .{ }^{13} \mathrm{C} \mathrm{NMR}$ $\left(75 \mathrm{MHz}, \mathrm{CDCl}_{3}\right): \delta=141.4,141.3,137.9,136.6,136.0,135.9\left(\mathrm{C}^{\text {quat }}\right), 130.4,129.9,126.4$, $126.2\left(C_{6} \mathrm{H}_{4}\right), 86.6\left(\mathrm{Fc}^{i p s o}\right), 69.3\left(C_{5} \mathrm{H}_{4}\right), 69.1\left(C_{5} \mathrm{H}_{5}\right), 68.2\left(C_{5} \mathrm{H}_{4}\right), 28.0\left(C_{2}\right), 15.8,15.7$, $15.5\left(\mathrm{CH}_{3}\right)$. MS (EI, $\left.70 \mathrm{eV}\right) \mathrm{m} / z: 484[\mathrm{M}]^{+}, 419[\mathrm{M}-\mathrm{Cp}]^{+}, 121[\mathrm{FeCp}]^{+}$. Elemental Anal. Calc. for $\mathrm{C}_{28} \mathrm{H}_{28} \mathrm{FeS}_{2}$ : C, 69.41; H, 5.83; S, 13.24. Found: C, 69.35; H, 5.75; S, $13.23 \%$. m.p. $=118-120^{\circ} \mathrm{C}$.

\subsection{X-ray crystal structure of compound 8}

A stable orange plate of $0.41 \times 0.32 \times 0.19 \mathrm{~mm}^{3}$ was mounted directly into the coldstream of an Oxford Cryostream crystal cooling apparatus installed on a STOE IPDS-II two-circle diffractometer, using perfluoropolyether oil. Data were collected using the $\omega$-scan technique. An empirical absorption correction was performed using the MULABS option [34] in PLATON [35]. The structure was solved by direct methods [36] and refined based on $F^{2}$ using the SHELXL-97 program [37]. All atoms were refined with anisotropic displacement parameters. Hydrogen atoms were placed in idealized positions and allowed to ride on the relevant carbon atom.

Crystallographic data for 8: $\mathrm{C}_{28} \mathrm{H}_{28} \mathrm{FeS}_{2}$; triclinic, $P \overline{1}, a=7.6480(4) \AA$, $b=9.4223(6) \AA$, $c=17.491(1) \AA, \quad \alpha=103.537(5)^{\circ}, \quad \beta=90.396(5)^{\circ}, \quad \gamma=107.989(5)^{\circ} ; \lambda=0.71073 \AA ; Z=2$; Number of reflections total/unique/observed $=33343 / 5079 / 4639 \quad(2 \theta=54.26) ; \quad 284$ parameters; $R_{1}, w R_{2}$ (all data) $=0.0311,0.0725$.

\subsection{Biochemical experiments}

Procedures for the determination of the relative binding affinity (RBA) for ER $\alpha$ and ER $\beta$ and cell culture conditions have been previously reported [38]. 


\section{Results and discussion}

\subsection{Synthesis}

Compounds $\mathbf{4}$ and $\mathbf{8}$ were synthesized by McMurry cross-coupling reactions between propionyl ferrocene and 4,4'-bis(t-butylthio)benzophenone or 4,4'bis(methylthio)benzophenone, respectively, as shown in Scheme 1. The reaction of the $t$ butylthioether 4 with an excess of $\mathrm{BBr}_{3}$ and acetyl chloride gave us the expected thioacetyl 6 in moderate yield. Although this deprotection/acetylation process proceeded smoothly in the case of $\mathbf{4}$, the methylthioether $\mathbf{8}$ was unreactive under these conditions. Even a mixture of $\mathrm{HNEt}_{2} / \mathrm{NaH} / \mathrm{HMPT}$, which has been described as a dealkylation reagent for alkyl aryl sulfides [39], was not strong enough to demethylate 8 .
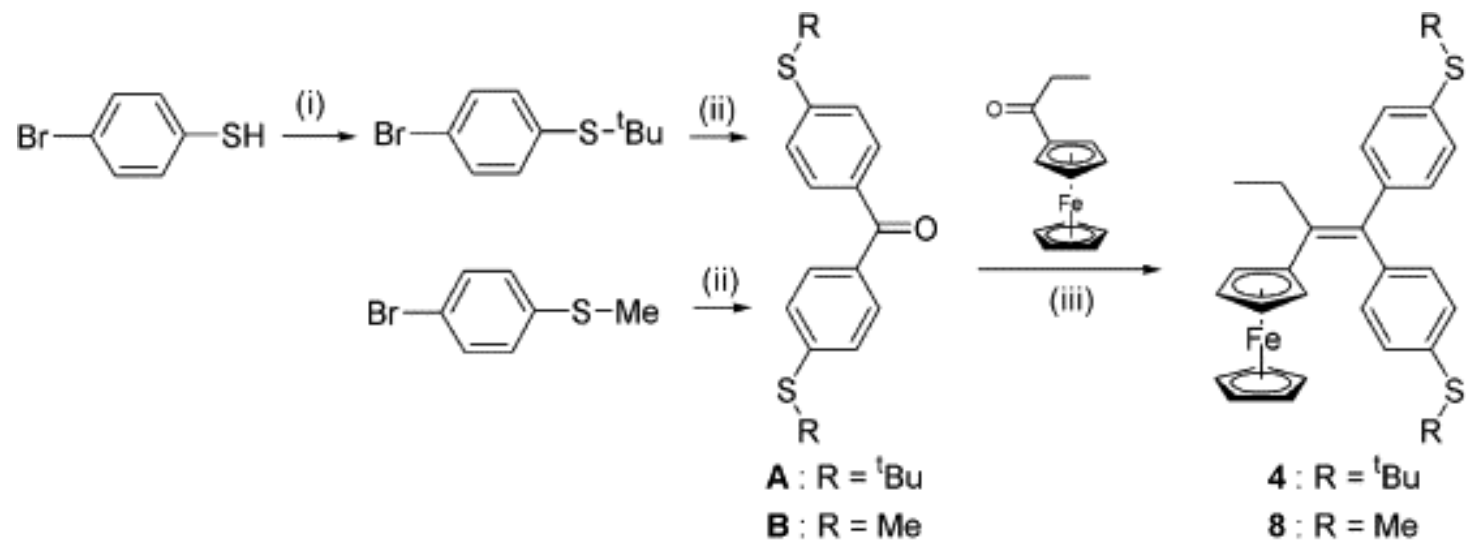

$4: \mathrm{R}={ }^{\mathrm{I}} \mathrm{Bu}$

B : $R=M e$

8: $R=M e$

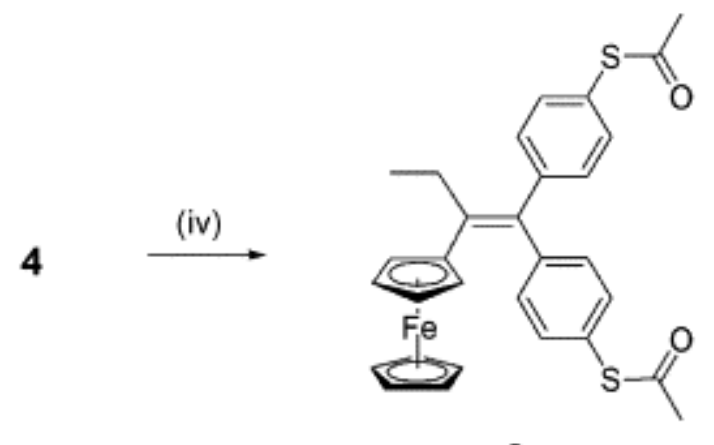

6

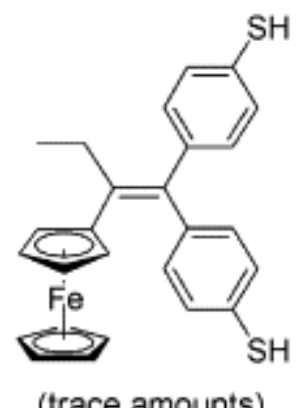

Scheme 1. (i) $1-{ }^{t} \mathrm{BuCl}, \mathrm{AlCl}_{3}$, r.t., $1 \mathrm{~h}$ and $2-\mathrm{H}_{2} \mathrm{O}$; (ii) $1-{ }^{n} \mathrm{BuLi} \mathrm{Et}_{2} \mathrm{O}-30{ }^{\circ} \mathrm{C} \rightarrow-10{ }^{\circ} \mathrm{C}, 3 \mathrm{~h}, 2-$ $\mathrm{Me}_{2} \mathrm{NC}(\mathrm{O}) \mathrm{Cl}, \mathrm{Et}_{2} \mathrm{O},-50{ }^{\circ} \mathrm{C} \rightarrow 0{ }^{\circ} \mathrm{C}, 3 \mathrm{~h}$, and $3-\mathrm{NH}_{4} \mathrm{Cl}, \mathrm{H}_{2} \mathrm{O}$; (iii) $1-\mathrm{TiCl}_{4} / \mathrm{Zn}$, THF, reflux, $3-6 \mathrm{~h}$ and $2-$ $\mathrm{HCl}, \mathrm{H}_{2} \mathrm{O}$; and (iv) 1 - exc. $\mathrm{BBr}_{3}$, exc. $\mathrm{H}_{3} \mathrm{CC}(\mathrm{O}) \mathrm{Cl}, \mathrm{CH}_{2} \mathrm{Cl}_{2}$, r.t., 6 days and $2-\mathrm{NaHCO}_{3(\mathrm{aq})}$.

We then attempted to deacetylate 6 to obtain the free thiol by reaction with aqueous $\mathrm{NH}_{4} \mathrm{OH}$. Although traces of the desired compound could be detected in the mass spectrum, the free 
thiol has not yet been isolated. Free aromatic thiols are known to be unstable to oxidation, and thus can be difficult to isolate [40].

Compound 7 was synthesized by McMurry cross-coupling reaction between propionyl ferrocene and 4,4'-dimethoxybenzophenone, as shown in Scheme 2. The acetoxy derivative 5 was synthesized by the reaction of the known compound $\mathbf{2}$ with an excess of acetyl chloride at ambient temperature.

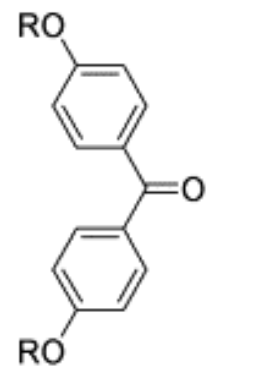

$\mathrm{R}=\mathrm{H}, \mathrm{Me}$
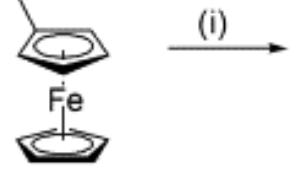

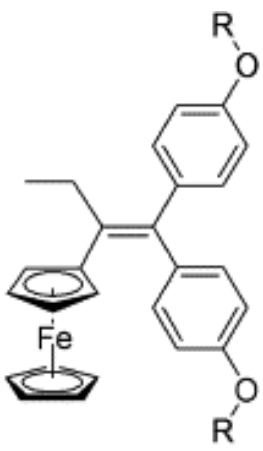

2: $\mathrm{R}=\mathrm{H}$

$7: \mathrm{R}=\mathrm{Me}$
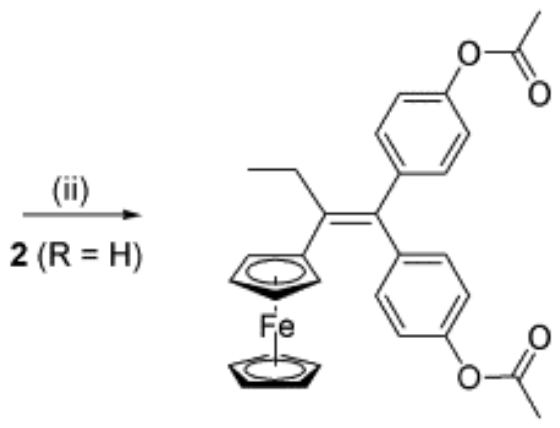

5

Scheme 2. (i) $1-\mathrm{TiCl}_{4} / \mathrm{Zn}$, THF, reflux, $3 \mathrm{~h}$ and $2-\mathrm{HCl}, \mathrm{H}_{2} \mathrm{O}$ and (ii) $1-$ exc. $\mathrm{H}_{3} \mathrm{CC}(\mathrm{O}) \mathrm{Cl}_{1} \mathrm{CH}_{2} \mathrm{Cl}_{2}$, r.t., $12 \mathrm{~h}$ and $2-\mathrm{H}_{2} \mathrm{O}$.

\subsection{X-ray crystal structure of 8}

The X-ray crystal structure of $\mathbf{8}$ is shown in Fig. 1. All atoms connected to the central double bond are located in a common plane (r.m.s. deviation $0.02 \AA$ ). The cyclopentadienyl ring attached to the double bond is almost coplanar with the latter plane (dihedral angle $3.7(1)^{\circ}$ ) whereas the two phenyl rings are almost perpendicular to that plane (dihedral angles $70.21(4)^{\circ}$ and $89.37(4)^{\circ}$ ). Whereas one of the thiomethyl groups (S2-C47) is almost coplanar (torsion angle $\mathrm{C} 47-\mathrm{S} 2-\mathrm{C} 44-\mathrm{C} 43-1.89(15)^{\circ}$ ) with the phenyl ring to which it is attached, the other one (S1-C37) is considerable twisted (torsion angle C37-S1-C34-C33 - 29.87(15) ${ }^{\circ}$ ) out of the plane of the aromatic ring to which it is attached. The two cyclopentadienyl rings are coplanar (dihedral angle $\left.2.05(14)^{\circ}\right)$. There are no short $\mathrm{C}-\mathrm{H} \cdots \mathrm{X}$ contacts. 


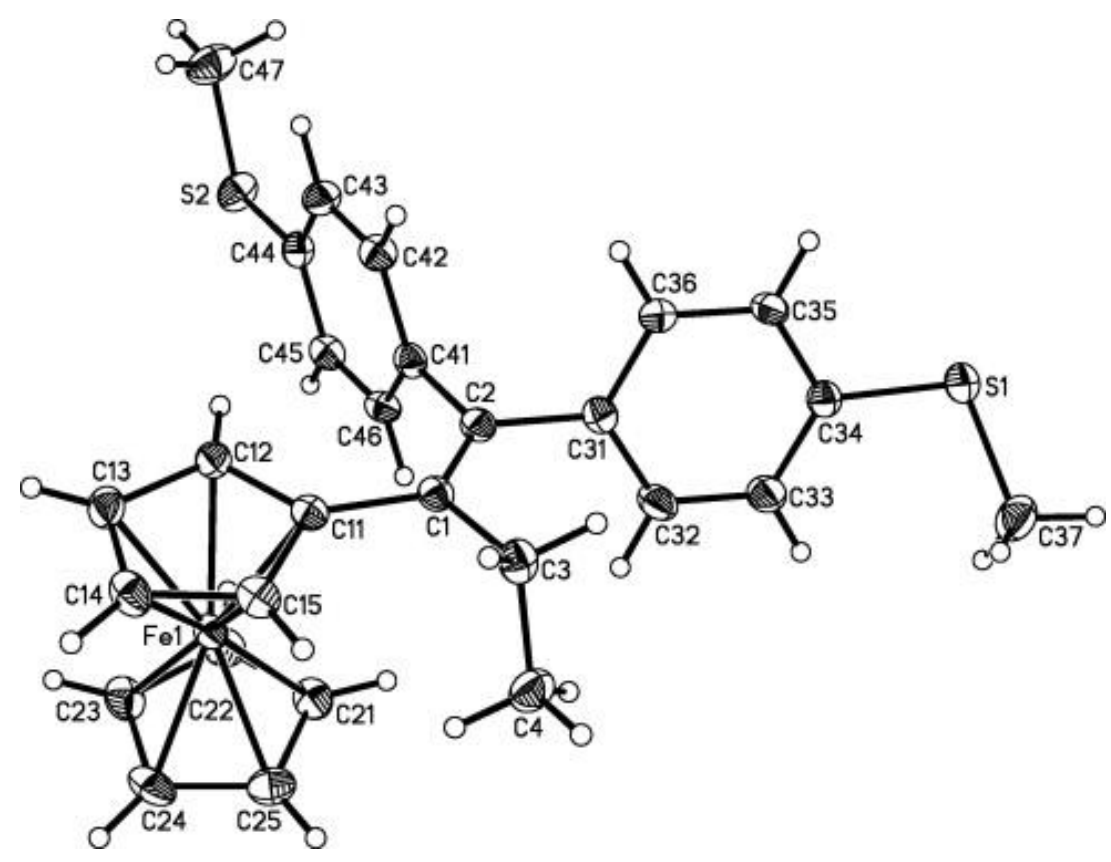

Fig. 1. ORTEP diagram of $\mathbf{8}$. Ellipsoids shown at 50\% probability.

\subsection{Biochemical results}

\subsubsection{Receptor affinity}

The affinities for the estrogen receptor of the newly synthesized complexes were measured on the two isoforms of the receptor, ER $\alpha$ and ER $\beta$, and are reported as relative binding affinity (RBA) values in Table 1. The RBA values for both isoforms of the receptor are very low for all of the compounds, except for $\mathbf{5}$, which showed a moderate affinity for ER $\alpha$. In general, the poor affinity can be explained by the fact that the hydroxyl groups are protected with methyl or acetyl groups in this series of molecules, thus preventing stabilizing bioligand-receptor hydrogen bonding interactions. It is also interesting to note that the affinity of the known ferrocene diphenol 2 for ER $\beta$ is much higher than for $E R \alpha$, while this trend is not observed with the new compounds 5-8. This indicates that neither $\mathbf{5}$ nor $\mathbf{7}$ were hydrolysed to $\mathbf{2}$ under the experimental conditions which involve purified ER instead of living cells. 
Table 1. Relative binding affinity (RBA) of the compounds for the two isoforms of the estrogen receptor $(\mathrm{ER} \alpha$ and $\mathrm{ER} \beta)$ and effect of $1 \mu \mathrm{M}(1 \mathrm{nM}$ of $17 \beta$-estradiol) on cell growth after 5 days of culture of hormone-dependent (MCF-7) and hormone-independent (MDA-MB-231) breast cancer cells

\begin{tabular}{|c|c|c|c|c|}
\hline \multirow{2}{*}{ Compound } & \multicolumn{2}{|l|}{$\operatorname{RBA}(\%)^{\mathrm{a}}$} & \multirow{2}{*}{$\operatorname{MCF}-7^{\mathrm{a}, \square}(\%)$} & \multirow{2}{*}{ MDA-MB-231 ${ }^{\mathrm{a}, *}(\%)$} \\
\hline & ERa & $\mathbf{E R} \boldsymbol{\beta}^{\mathbf{b}}$ & & \\
\hline $17 \beta$-Estradiol & $100^{\mathrm{c}, \mathrm{d}}$ & $100^{\mathrm{d}}$ & $222 \pm 8^{\mathrm{f}}$ & No effect \\
\hline 5 & $6.5 \pm 0.5^{\mathrm{c}}$ & $0.8 \pm 0.1$ & $37.7 \pm 2^{\mathrm{g}}$ & $22.5 \pm 0.9$ \\
\hline 6 & $0.270 \pm 0.005^{\mathrm{b}}$ & $0.03^{\mathrm{e}}$ & $187 \pm 32^{\mathrm{f}}$ & $99 \pm 5$ \\
\hline 7 & $0.20 \pm 0.1^{\mathrm{c}}$ & $0.26 \pm 0.02$ & $151 \pm 15^{\mathrm{f}}$ & $101 \pm 1$ \\
\hline 8 & $0.04 \pm 0.01^{\mathrm{c}}$ & $0.037 \pm 0.005$ & $169.5 \pm 20^{\mathrm{f}}$ & $99 \pm 4$ \\
\hline $2^{\mathrm{h}}$ & $9.6 \pm 0.6^{c}$ & $16.3 \pm 1.5$ & $37 \pm 5^{\mathrm{g}}$ & $29 \pm 1.6$ \\
\hline \multicolumn{5}{|c|}{ Values compared to control, defined as $100 \%$. } \\
\hline \multicolumn{5}{|c|}{ a Mean of two experiments \pm range. } \\
\hline \multicolumn{5}{|c|}{${ }^{\mathrm{b}}$ Purified receptor. } \\
\hline \multicolumn{5}{|c|}{ ' ${ }^{\mathrm{c}}$ Receptor from lamb uterine cytosol. } \\
\hline \multicolumn{5}{|c|}{${ }^{\mathrm{d}}$ Value by definition. } \\
\hline \multicolumn{5}{|c|}{${ }^{\mathrm{e}}$ One determination. } \\
\hline \multicolumn{5}{|c|}{ f Medium without phenol red. } \\
\hline${ }^{\mathrm{g}}$ Medium with & phenol red. & & & \\
\hline${ }^{\mathrm{h}}$ Values from & ef. [5]. & & & \\
\hline
\end{tabular}

\subsubsection{Effect of the compounds on the growth of breast cancer cells}

The effect of these complexes at a concentration of $1 \mu \mathrm{M}$ was studied on hormoneindependent (MDA-MB-231) and hormone-dependent (MCF-7) breast cancer cells and the results are displayed in Table 1. Any antiproliferative effect observed on the hormoneindependent breast cancer cells can be attributed only to a cytotoxic effect. On these cells, only compound 5 showed a antiproliferative effect, which is of comparable magnitude to that shown by the reference compound ferrocenyl diphenol $2 \quad\left(\operatorname{IC}_{50}(\mathbf{5})=0.5 \mu \mathrm{M}\right.$; $\left.\mathrm{IC}_{50}(2)=0.6 \mu \mathrm{M}\right)$. This strongly suggests that enzymes in the living cells hydrolysed the ester function of $\mathbf{5}$ to generate the dihydroxyl derivative $\mathbf{2}$ in situ, as observed in other systems such as ester-estrogen cleavage [41-43] and the activation of fluorescent probes [44]. Compound 2 can then engage in QM formation to give rise to cytotoxic effects. As thioesterases are also known to exist in breast cancer cells [45], one might therefore expect hydrolysis to proceed in 
the case of the analogous sulfur compound $\mathbf{6}$ as well. However, no antiproliferative effect was observed for $\mathbf{6}$, suggesting that the putative ferrocenyl thiophenol is not cytotoxic.

Neither of the ether compounds $\mathbf{7}$ nor $\mathbf{8}$ had any significant effect against the MDA-MB-231 cell line $\left(\mathrm{IC}_{50}(7)=82.5 \mu \mathrm{M}\right)$, likely because these compounds are simply not hydrolysed in situ. Eukaryotic etherase enzymes have been detected in liver cells [46] but not yet in breast cancer cells, and thus hydrolysis of the methyl protected compounds does not proceed in vitro. Finally, as expected, estradiol has no effect on these cells lacking ER $\alpha$ (see Table 1).

At $1 \mu \mathrm{M}$, all of the compounds show a strong proliferative effect on the hormone-dependent breast cancer cell line MCF-7, except compound 5, which shows an antiproliferative effect. Again, this antiproliferative effect is similar to that of previously reported compound $\mathbf{2}$, suggesting in situ hydrolysis and possible QM formation. The strong estrogenic effects exhibited by 6-8 are surprising, considering the low affinity the compounds have for ER $\alpha$. However, we have already noticed that some compounds with low RBA values are estrogenic at this high concentration [38].

\section{Conclusions}

We have synthesized four new ferrocene compounds containing protected phenol and thiophenol functions to determine how the replacement of an oxygen atom by a sulfur atom and the presence of two types of protecting groups influence the compounds' proliferative/antiproliferative effects against breast cancer. Compounds 6-8 acted as pure estrogens, while compound $\mathbf{5}$ showed cytotoxic effects similar in magnitude to those observed with previously described ferrocenyl diphenol $\mathbf{2}$. These results suggest that the thiophenol analogue of $\mathbf{6}$ is non-cytotoxic, further implicating the production of QM in the cytotoxicity of 2 and related compounds.

\section{Acknowledgements}

We thank A. Cordaville for technical assistance, and the Agence Nationale de la Recherche for financial support (No. ANR-06-BLAN-0384-01, "FerVect"). J.B.H. acknowledges a fellowship within the Postdoc Programme of the German Academic Exchange Service (DAAD). 


\section{Appendix A. Supplementary material}

CCDC 665513 contains the supplementary crystallographic data for this paper. These data can be obtained free of charge from The Cambridge Crystallographic Data Centre via www.ccdc.cam.ac.uk/data_request/cif. Supplementary data associated with this article can be found, in the online version, at doi:10.1016/j.jorganchem.2007.12.011.

\section{References}

[1] G. Jaouen, Bioorganometallics, Wiley-VCH, Weinheim, Germany, 2006.

[2] D. Dive, C. Biot, ChemMedChem (2007), doi:10.1002/cmdc. 200700127.

[3] M. Melchart, P.J. Sadler, in: G. Jaouen (Ed.), Bioorganometallics, Wiley-VCH, 2005, p. 39.

[4] A. Vessières, S. Top, W. Beck, E.A. Hillard, G. Jaouen, Dalton Trans. 4 (2006) 529.

[5] A. Vessières, S. Top, P. Pigeon, E.A. Hillard, L. Boubeker, D. Spera, G. Jaouen, J. Med. Chem. 48 (2005) 3937.

[6] S. Top, A. Vessières, G. Leclercq, J. Quivy, J. Tang, J. Vaissermann, M. Huché, G. Jaouen, Chem. Eur. J. 9 (2003) 5223.

[7] G. Jaouen, S. Top, A. Vessières, G. Leclercq, M.J. McGlinchey, Curr. Med. Chem. 11 (2004) 2505.

[8] E.A. Hillard, A. Vessières, L. Thouin, G. Jaouen, C. Amatore, Angew. Chem., Int. Ed. 45 (2006) 285.

[9] M. Kurihara, H. Sano, M. Murata, H. Nishihara, Inorg. Chem. 40 (2001) 4.

[10] N.N. Meleshonkova, D.B. Shpakovsky, A.V. Fionov, A.V. Dolganov, T.V. Magdesieva, E.R. Milaeva, J. Organomet. Chem. 692 (2007) 5339.

[11] S. Top, A. Vessières, C. Cabestaing, I. Laios, G. Leclercq, C. Provot, G. Jaouen, J. Organomet. Chem. 637 (2001) 500.

[12] A. Zhou, S. Kikandi, O.A. Sadik, Electrochem. Commun. 9 (2007) 2246. 
[13] I.M.C.M. Rietjens, M.G. Boersma, H.v.d. Woude, S.M.F. Jeurissen, M.E. Schutte, G.M. Alink, Mutat. Res. 574 (2005) 124.

[14] T.J. Monks, D.C. Jones, Curr. Drug Metabol. 3 (2002) 425.

[15] H. Liu, Z. Qin, G.R.J. Thatcher, J.L. Bolton, Chem. Res. Toxicol. (2007), doi:10.1021/tx7001367.

[16] P.W. Fan, F. Zhang, J.L. Bolton, Chem. Res. Toxicol. 13 (2000) 45.

[17] L. Yu, H. Liu, W. Li, F. Zhang, C. Luckie, R.B. van Breemen, G.R. Thatcher, J.L. Bolton, Chem. Res. Toxicol. 17 (2004) 879.

[18] J.L. Bolton, E. Pisha, F. Zhang, S. Qiu, Chem. Res. Toxicol. 11 (1998).

[19] J.P. Driscoll, K. Kornecki, J.P. Wolkowski, L. Chupak, A.S. Kalgutkar, J.P. O’Donnell, Chem. Res. Toxicol. 20 (2007) 1488.

[20] M. Di Antonio, F. Doria, M. Mella, D. Merli, A. Profumo, M. Freccero, J. Org. Chem. 72 (2007) 8354.

[21] N. Hulsman, J.P. Medema, C. Bos, A. Jongejan, R. Leurs, M.J. Smit, I.J.P. de Esch, D. Richel, M. Wijtmans, J. Med. Chem. 50 (2007) 2424.

[22] G. Gaudiano, T.H. Koch, Chem. Res. Toxicol. 4 (1991) 2.

[23] T. Itoh, K. Fujikawa, M. Kubo, J. Org. Chem. 61 (1996) 8329.

[24] U. Burner, C. Obinger, FEBS Lett. 411 (1997) 269.

[25] C. Asche, Mini-Rev. Med. Chem. 5 (2005) 449.

[26] D. Osella, M. Ferrali, P. Zanello, F. Laschi, M. Fontani, C. Nervi, G. Cavigiolio, Inorg. Chim. Acta 306 (2000) 42.

[27] P. Amrolia, S.G. Sullivan, A. Stern, R. Munday, J. Appl. Toxicol. 9 (1989) 113.

[28] E. von Angerer, The Estrogen Receptor as a Target for Rational Drug Design, Springer-Verlag, Heidelberg, Germany, 1995.

[29] A. Stañczak, A. Ferra, Pharmacol. Rep. 58 (2006) 599.

[30] T. Pinault, F. Chérioux, B. Therrien, G. Süss-Fink, Heteroatom Chem. 15 (2004) 121.

[31] S. Top, B. Dauer, J. Vaissermann, G. Jaouen, J. Organomet. Chem. 541 (1997) 355. 
[32] P. Lucas, N.E. Mehdi, H.A. Ho, D. Bélanger, L. Breau, Synthesis 9 (2000) 1253.

[33] S. Top, J. Tang, A. Vessières, D. Carrez, C. Provot, G. Jaouen, Chem. Commun. (1996) 955.

[34] R.H. Blessing, Acta Crystallogr., Sect. A 51 (1995) 33.

[35] A.L. Spek, J. Appl. Cryst. 36 (2003) 7.

[36] G.M. Sheldrick, Acta Crystallogr., Sect. A 46 (1990) 467.

[37] G.M. Sheldrick, SHELX-97 Program for the Refinement of Crystal Structures, University of Göttingen, Germany, 1997.

[38] E.A. Hillard, P. Pigeon, A. Vessières, C. Amatore, G. Jaouen, Dalton Trans. (2007) 5073 .

[39] S. Cabiddu, S. Melis, P.P. Piras, F. Sotgiu, Synthesis 7 (1982) 583.

[40] J.M. Tour, L.R. Jones II, D.L. Pearson, J.J.S. Lamba, T.P. Burgin, G.M. Whitesides, D.L. Allara, A.N. Parikh, S.V. Atre, J. Am. Chem. Soc. 117 (1995) 9529.

[41] J. Katz, T.H. Finlay, S. Banerjee, M. Levitz, J. Steroid Biochem. 26 (1987) 687.

[42] J. Katz, M. Levitz, S.S. Kadner, T.H. Finlay, J. Steroid Biochem. Molec. Biol. 38 (1991) 17.

[43] K.R. Barnes, A. Kutikov, S.J. Lippard, Chem. Biol. 11 (2004) 557.

[44] G. Bartosz, Clin. Chim. Acta 368 (2006) 53.

[45] S. Smith, D. Pasco, J. Pawlak, B. Thompson, M. Stampfer, S. Nandi, J. Natl. Cancer Inst. 73 (1984) 323.

[46] J. Axelrod, Biochem. J. 63 (1956) 634. 